\title{
Zeitgenössisches zu den Auswirkungen der „68er-Bewegung“ auf das Arbeitsrecht
}

2008 ist in den Zeitungen viel über die vierzig Jahre zurückliegenden Ereignisse des Jahres 1968 und die damalige „Außerparlamentarische Opposition“ (APO) geschrieben worden. Meist waren es Zeitgenossen, die sich aus der Distanz erinnerten und große Epochen-Bilder entwarfen. Immer wieder ging es dabei aber um die Frage, ob jenes Jahr rückblickend als Zäsur oder bloß als Modifikation durchdringender Kontinuität der (deutschen) Nachkriegszeit einzuschätzen sei. Das „Internationale Max-PlanckForschungskolleg für vergleichende Rechtsgeschichte" hat diese Frage auch einer Reihe von Juristinnen und Juristen gestellt, die sie als Zeitgenossen für die Entwicklung von Rechtswissenschaft und Rechtspraxis beantworten sollten. Meine ganz subjektiven Erlebnisse und Antworten zum Arbeitsrecht sind nur als Anregung zu einem intensiveren ,Diskurs“ gedacht.

\section{A. Der historische Blick des, „Zeitzeugen “im Arbeitsrecht}

Wenn ich mich recht erinnere, war es der 3. Juni 1967, als ich in meinem FIAT 500 voller Bücher von Berlin nach Gießen fuhr, um dort als wissenschaftliche Hilfskraft am Lehrstuhl von Helmut Ridder als Referendar zu arbeiten. Ich war 24 Jahre alt und hatte - obwohl nicht mehr Student - am Vortage an der großen Anti-Schah-Demonstration teilgenommen, die mit dem Tod Benno Ohnesorgs endete. Sowohl mein Auto wie auch ich selbst hatten dabei einige Beulen davongetragen. Ich war nicht wirklich unglücklich, dem Berliner Hexenkessel zu entkommen und in die friedliche hessische Provinz zu ziehen.

Dennoch empfand ich mich als Teil der „Außerparlamentarischen Opposition“ und ihres Widerstands gegen den Vietnam-Krieg und gegen die Notstandsgesetze. Gerade deshalb war Helmut Ridder während einer Vortragsreihe auf mich aufmerksam geworden. Mein Interesse galt dem Völkerrecht und vor allem dem Verfassungsrecht. Das Arbeitsrecht erschien eher uninteressant. Es erschöpfte sich an den damaligen Universitäten fast durchweg im Vertragsrecht des BGB - während seine dem Verfassungsrecht zugewandte Seite, nämlich das Arbeitskampfrecht, fast unbeleuchtet blieb. An den großen Streit um das Betriebsverfassungsrecht von 1952 mit den Gutachten von Nipperdey und Abendroth zum politischen Streik und zum Sozialstaatsprinzip erinnerte keiner mehr. Stattdessen galt das Verdikt des Großen BAG-Senats von 1955: „Arbeitskämpfe (Streik und Aussperrung) sind im allgemeinen unerwünscht, da sie volkswirtschaftliche Schäden mit sich bringen und den im Interesse der Gesamtheit liegenden sozialen Frieden beeinträchtigen...". Die Gewerkschaften waren dadurch als Störenfriede in unbehagliche Nähe zum Sozialismus gerückt, obwohl sie sich doch schon

1 BAG (v. 28.1.1955) AP Nr. 1 zu Art. 9 GG Arbeitskampf. 
seit dem DGB-Aktionsprogramm von 1955 durchaus marktwirtschaftlich orientiert hatten.

Nur Thilo Ramm hatte schon 1961 „Die Parteien des Tarifvertrages“ als solche in den Blick genommen und 1964 die Sozialadäquanztheorie Nipperdeys mit ihren nationalsozialistischen Bezügen bloßgestellt. ${ }^{2}$ In den juristischen Fakultäten waren es vor allem solche Kontinuitäten in der Professorenschaft, welche auch die eher unpolitisch gestimmten Jurastudenten gegen die alte „Ordinarienuniversität“ aufbrachten, zumal sie in praktisch allen Rechtsgebieten nachweisbar waren. Vorlesungsboykott und selbstorganisierte kritische Gegenveranstaltungen trieben die Studierenden an die Seite der Politologen und Soziologen, welche in ,Sit-ins' und Demonstrationen einen radikalen gesellschaftlichen Neubeginn der als faschistisch empfundenen alten Bundesrepublik forderten.

Im Zentrum der großen Aktionen stand freilich zunächst nicht das Arbeitsrecht, sondern das Verfassungsrecht. Die von der großen Koalition geplanten Notstandsgesetze sah man als Versuch der Wiedereinführung des Art. 48 WRV in neuem Gewand, d.h. als Ermächtigung der Exekutive zu antiparlamentarischen und antidemokratischen Maßnahmen, mit denen Hindenburg, Papen und Schleicher unter juristischer Mithilfe von Carl Schmitt schon die Weimarer Republik zerstört hatten. Zentrum der Anti-Notstandsbewegung war das „Kuratorium Notstand der Demokratie“, in dem sich Wissenschaftler, Gewerkschafter und Studenten vereinten, die äußerst öffentlichkeitswirksame Massenveranstaltungen organisierten. Da Helmut Ridder zu den führenden Köpfen des Kuratoriums zählte, konnte ich dies alles aus nächster Nähe miterleben. Ridder vermittelte uns nicht nur das Bewusstsein persönlichen Engagements, sondern auch eine kritische wissenschaftliche Perspektive aus historischer Analyse. Für uns aber stand wohl das Erlebnis einer rauschenden Öffentlichkeit und als Ziel die Demokratisierung der politisch agierenden Gesellschaft im Vordergrund.

Vor allem kam man dabei auch in Kontakt mit den „,verbündeten“ Teilen der Gewerkschaften. Schon 1960 und 1966 in den BAG-Verfahren über die gewerkschaftliche Urabstimmung ${ }^{3}$ als „Kampfmaßnahme“ und über die Zulässigkeit tariflicher Differenzierungsklauseln ${ }^{4}$ waren von Gamillscheg ${ }^{5}$ sowie von Ridder ${ }^{6}$ und Ramm ${ }^{7}$ die Bedeutung der Gewerkschaften für die Funktionsfähigkeit des Sozialstaats und die öffentliche Willensbildung gutachterlich herausgestellt worden. Zwar blieb das beide Male gegen die h.M. erfolglos, doch schien nunmehr die Probe aufs Exempel gekommen. 1968 propagierten die radikalen Teile der außerparlamentarischen Opposition außerhalb des „Kuratoriums“ nämlich bereits die sozialistische Revolution im Schulterschluss mit der „Arbeiterbewegung“. Halbwegs legalistisch Gesinnte wollten wenigstens aus dem neuen Widerstandsrecht des Art. 20 Abs. 4 GG, der als Konzession an die Gewerkschaften gedacht war, ein Recht auf Generalstreiks ableiten. Ich selbst ha-

2 Der Streik als unerlaubte Handlung, AuR 1964, 353.

3 BAG (v. 31.10.1958) AP Nr. 2 zu § 1 TVG Friedenspflicht.

4 BAG (v. 29.11.1967) AP Nr. 13 zu Art. 9 GG; vgl. auch BAG (v. 9.5.2007) DB 61 (2008), 358.

5 Franz Gamillscheg, Die Differenzierung nach der Gewerkschaftszugehörigkeit, Berlin 1966.

6 Helmut Ridder, Gestattet das Grundgesetz die Erhebung sogenannter Solidaritätsbeiträge?, Bonn 1961.

7 Thilo Ramm, Der Arbeitskampf und die Gesellschaftsordnung des Grundgesetzes, Stuttgart 1965. 
be das damals als verfassungswidrig und unhistorisch abgelehnt. ${ }^{8}$ Mir scheint unverändert, dass die rechtsgeschichtliche und die verfassungsrechtliche Perspektive uns einen eigensinnigen Abstand zum juristischen Zeitgeschehen vermitteln. Das gilt auch dann, wenn man die arbeitsrechtlichen Entwicklungen als Zeitzeuge beschreiben soll.

\section{B. Arbeitsrechtliche Grundpositionen aus der 68er-Phase}

Am 1. Mai 1968 gab es eine riesige Demonstration gegen die Notstandsgesetze im Bonner Hofgarten, an der mehr als 50.000 Studenten, Gewerkschafter, Lehrer und Schüler teilnahmen. Am 24. Juni wurde die Notstandsverfassung dann doch von der großen Koalition verabschiedet. Neben dem Widerstandsrecht gab es einige weitere Konzessionen an die Gewerkschaften. Dazu gehörte vor allem der Schutz der Arbeitskämpfe vor staatlichen Eingriffen in bestimmten Notstandsfällen nach dem neuen Art. 9 Abs. 3 Satz 3 GG. Diese Regelung, welche u.a. auf eine Initiative des SPD-Abgeordneten Matthöfer aus der IG Metall zurückging, spielt in der arbeitskampfrechtlichen Diskussion bis heute eine Rolle. Sie hat seinerzeit jedoch dazu geführt, dass sich die Gewerkschaften bis auf ihre ganz linken Flügel aus den Linien der APO zurückzogen. Förmliche Unterstützungsbeschlüsse der Gewerkschaftsvorstände existierten ohnehin nicht.

Tatsächlich waren ihre Organisationen schon seit 1967 über das sog. Stabilitätsgesetz und die „Konzertierte Aktion“ in die wirtschaftliche Globalsteuerung der Bundesregierung eingebunden. Sie praktizierten eine keynesianisch motivierte Tarifpolitik, welche bis 1969 den Rückgang des zuvor perhorreszierten Preisanstiegs bewirkte. Das Modell des erfolgreichen tripartistischen Verbändestaats hatte die Gewerkschaften hoffähig gemacht. Die intellektuellen Analytiker von 1968 erkannten in diesem „Spätkapitalismus“ ein politisches System, welches in der Lage war, seine „Legitimationsprobleme“ zu lösen, nämlich den „kapitalistischen Grundwiderspruch“ zwischen „fortschreitender Vergesellschaftung der Produktion“ und profitorientierter Disposition über das „Produktionsergebnis“.9

Die Septemberstreiks von 1969 bei den Ford-Werken und in vielen Metallbetrieben zeigten indessen die Grenzen der Legitimationskraft der IG-Metall im „Spätkapitalismus" auf, weil die streikenden Arbeiter gegen die Gewerkschaft einen Lohnnachschlag verlangten, der ihnen auf Grund der zurückhaltenden Tarifabschlüsse versagt bleiben musste. Zwar band die IG-Metall ihre Mitglieder wieder ein, indem sie deren Forderungen aufnahm und später durchsetzte, doch waren die Grenzen des Legitimationskonzepts durch diese ,,wilden“ Streiks deutlich geworden. ${ }^{10}$

Hieran schieden sich jetzt die Geister. Die „Linken“ sahen das von ihnen als „Ordnungsmacht"-Ideologie verteufelte Konzept der konzertierten Tarifpolitik als völlig gescheitert an. Sie propagierten stattdessen den Kampf der Arbeiterklasse gegen das Sys-

8 Otto Ernst Kempen, Widerstandsrecht (Art. 24 Abs. 4 GG), in: Dieter Sterzel (Hg.), Kritik der Notstandsgesetze, Frankfurt 1968, 65 ff., 83 ff.

9 Claus Offe, Spätkapitalismus - Versuch einer Begriffsbestimmung, in: Ders., Strukturprobleme des kapitalistischen Staates. Aufsätze zur politischen Soziologie, Frankfurt 1972, 7, 23.

10 Vgl. Michael Kittner, Arbeitskampf. Geschichte, Recht, Gegenwart, München 2005, 684. 
tem des „organisierten Kapitalismus“. In der sozialen Marktwirtschaft sahen sie einen ,octroi' der amerikanischen Besatzungsmacht gegenüber den einstmals sozialisierungsbereiten Westdeutschen. ${ }^{11}$ Ihn gelte es nach dem Vorbild des ,großen englischen Dockarbeiterstreiks“" von 1972 klassenkämpferisch zu beseitigen. ${ }^{12}$

Mochte dies auch an entsprechende Vorstellungen der 68er-Bewegung anknüpfen, so hatte die Idee von Arbeitsrecht als Klassenkampf jetzt dennoch keine Chance mehr.

Die Wahl Willy Brandts zum Bundeskanzler einer sozialliberalen Koalition mit dem Motto „Mehr Demokratie wagen“ hatte 1969 nämlich den Großteil der APO-Unterstützer wieder in die institutionelle Politik zurückgeholt. Diese „Realisten“ hatten den Schock der Septemberstreiks durchaus verspürt. Anders als die Linken sahen sie die Gewerkschaften als „Gegenmacht“ innerhalb der Marktwirtschaft des Sozialstaats. Durch sozialstaatlich inspirierte Gesetzgebung sollte der individuelle Schutz der Arbeitnehmer verbessert werden. Die Einführung einer Unternehmensmitbestimmung in allen Großunternehmen sollte die Beschäftigten an einer neuen sozialen Unternehmensverfassung beteiligen. Daneben galt es, die innergewerkschaftliche Demokratie $\mathrm{zu}$ verbessern. ${ }^{13}$ Schließlich sollte die verkrustete Arbeitskampf-Rechtsprechung des BAG zu Gunsten der Gewerkschaften aufgebrochen werden.

Alle diese Vorhaben wären ohne die 68er-Bewegung kaum realistisch gewesen. Sie brachen sich jetzt in SPD und FDP Bahn und mündeten in konkrete sozialstaatliche Projekte.

\section{Das Arbeitsrecht während der sozial-liberalen Koalition}

Ich selbst war 1969 als Wissenschaftlicher Assistent am Lehrstuhl von Christoph Sasse in Gießen mit dem Europäischen Wirtschaftsrecht, der Parlamentarisierung der Gemeinschaft und der Europäischen Aktiengesellschaft beschäftigt. Ende 1973 wurde ich Justitiar der damaligen IG Bau-Steine-Erden, denn ich wollte die Praxis der Gewerkschaften in ihrer Tarifpolitik und in ihrer wirtschaftlichen Globalsteuerung kennenlernen. Mich überraschten die pragmatisch-mitgliederorientierte Strategie und eine breit gefächerte tarifliche Sozialpolitik, die auch eigenständige Alterssicherungstarifsysteme umfasste. Auffällig war die enge Verbindung zur Bundesregierung bei allen einschlägigen Gesetzgebungsvorhaben. Sie führte gelegentlich dazu, dass besonders innovative Tarifverträge in entsprechende Gesetze umgesetzt wurden, z.B. das Konkursausfallgeld, heute Insolvenzgeld, nach $\S \S 183$ ff. SGB III.

11 Eberhard Schmidt, Die verhinderte Neuordnung 1945-1952. Zur Auseinandersetzung um die Demokratisierung der Wirtschaft in den westlichen Besatzungszonen und in der Bundesrepublik Deutschland, Frankfurt 1970; Ute Schmidt/Tilman Fichter, Der erzwungene Kapitalismus, Klassenkämpfe in den Westzonen 1945-1948, Berlin 1971.

12 Ulrich Mückenberger, Arbeitsrecht und Klassenkampf. Der große englische Dockarbeiterstreik 1972 (Theorie und Praxis der Gewerkschaften), Frankfurt 1974.

13 Horst Föhr, Anforderungen des Grundgesetzes an den Aufbau von Verbänden, NJW 28 (1975), 617 ff.; Gunther Teubner, Organisationsdemokratie und Verbandsverfassung. Rechtsmodelle für politisch relevante Verbände (Tübinger rechtswissenschaftliche Abhandlungen 47), Tübingen 1978. 
Noch die große Koalition hatte - ebenfalls auf Grund tariflicher Vorbilder - die Lohnfortzahlung im Krankheitsfall für Arbeiter (1969) eingeführt. Jetzt folgten der Betriebsübergang ( $\$ 613$ a BGB), das Schwerbehindertengesetz, das Gesetz zur Verbesserung der betrieblichen Alterssicherung, das Bundesdatenschutzgesetz (1972) sowie das hoch umstrittene Gesetz über die Unternehmensmitbestimmung (1976).

Ihm ist eine heftige Diskussion vorausgegangen, an der sich vor allem damals jüngere Wissenschaftler wie Biedenkopf, Däubler, Scholz und Raiser beteiligt hatten. Hier, wie auch bei der Betriebsverfassung, lag der Schwerpunkt auf einem neuen Verständnis des Sozialstaatsprinzips und der Grundrechte als gesamtgesellschaftlich verstandener Werteordnung. Rechtshistorisch wurde die Mitbestimmung als Sozialisierungsalternative herausgearbeitet - während die Gewerkschaften etwas platt, aber durchaus auch in der 68er-Tradition, von der „Demokratisierung“ der Betriebe sprachen.

Das Ergebnis brachte dann bekanntlich keine wirkliche Parität zwischen Anteilseignern und Arbeitnehmern, sondern das Letztentscheidungsrecht des AR-Vorsitzenden. Dennoch erhoben 1976 die Arbeitgeber ihre gutachtengestützte Verfassungsbeschwerde. Sie veranlasste die IG-Metall zum Austritt aus der konzertierten Aktion, wodurch auch das Ende der stabilitätsgesetzlichen Globalsteuerung eingeläutet war. Bekanntlich bestätigte das BVerfG das Mitbestimmungsgesetz, ${ }^{14}$ welches von Kübler, Schmidt und Simitis ${ }^{15}$ verteidigt worden war. Die Gewerkschaften haben seine Umsetzung später durchweg - entgegen ihrer Demokratisierungs-Suada - sehr geschäftsmäßig betrieben. Inhaltlich blieb es bis heute den Gesellschaftsrechtlern überlassen. Sie haben die Ansätze zur Schaffung eines Unternehmensverfassungsrechts konsequent verhindert.

Fast könnte es scheinen, als sei die Aufbruchs- und Reformbewegung nach 1969 im Arbeitsrecht gänzlich in sozialliberale Gesetze geronnen. Tatsächlich sind aber auch die Tarifverträge ein wesentlicher Bestandteil arbeitsrechtlicher Regelungen. Auch hier gab es nach 1969 ganz neue Aktivitäten einer jüngeren Gewerkschaftsgeneration, die von Arbeitsrechtlern wie Detlef Hensche und Michael Kittner oder Friedhelm Farthmann unterstützt wurden. Eine neue innovative Tarifpolitik regulierte bewusst den Arbeitsmarkt durch Arbeitszeitverkürzung und milderte die Folgen der aufkommenden Informationstechnologie durch Rationalisierungsschutz-Abkommen. Wegen des Widerstands der Arbeitgeber mussten sie häufiger als früher mit Streiks durchgesetzt werden. Über ihnen hing freilich noch immer das 1955 und 1971 vom BAG scharf geschliffene Schwert der Aussperrung. Deshalb organisierte Michael Kittner für die IGMetall 1973 eine große wissenschaftliche Veranstaltung über „Streik und Aussperrung“, ${ }^{16}$ die zu einer repräsentativen Heerschau der Aussperrungskritiker wurde. Das dort geforderte Aussperrungsverbot kam zwar nie zustande. Dennoch hat das BAG 1980 nur noch die suspendierende Abwehraussperrung als eine durch den Verhältnis-

14 BVerfGE (v. 1.3.1979) 50, 290.

15 Friedrich Kübler/Walter Schmidt/Spiros Simitis, Mitbestimmung als gesetzgebungspolitische Aufgabe. Zur Verfassungsmäßigkeit des Mitbestimmungsgesetzes 1976, Baden-Baden 1978.

16 Vgl. Michael Kittner (Hg.), Streik und Aussperrung. Protokoll der wissenschaftlichen Veranstaltung der Industriegewerkschaft Metall vom 13. bis 15.9.1973 (Schriftenreihe der OttoBrenner-Stiftung 3), Frankfurt 1973. 
mäßigkeitsgrundsatz begrenzte Maßnahme zugelassen. ${ }^{17}$ Die umfangreiche Begründung dazu zeigt, dass jene neuartige Gemeinschaftsveranstaltung von Gewerkschaft und Wissenschaft aus dem Geiste von 1968 heraus nicht wirkungslos geblieben war.

\section{Die langfristig tätigen Akteure im Arbeitsrecht}

Der Zusammenbruch des dollargestützten Währungssystems von Bretton Woods und die seit 1973 frei floatenden Wechselkurse bewirkten eine rasche Globalisierung des internationalen Geldmarkts, welche die wirtschaftliche Globalsteuerung in Deutschland aushebelte und 1982 letztlich zur Regierungsübernahme durch Helmut Kohl geführt hat. Er wollte die Beschäftigung durch den Abbau arbeitsrechtlicher Schutzvorschriften fördern und lockerte deshalb den Kündigungsschutz durch das Beschäftigungsförderungsgesetz von 1985. Obwohl ansonsten nicht viel verändert wurde, blieb es in der Kritik der Gewerkschaften und der ,progressiven“ Arbeitsrechtler. Aber um wen handelte es sich dabei eigentlich?

Die rauschende Öffentlichkeit des Jahres 1968 mit den großen Stimmen von Adorno und Habermas, Abendroth und Ridder, Dutschke und Krahl, Jens und Marcuse war längst verhallt. Andererseits gab es gerade im Arbeitsrecht zahlreiche Diskussionszirkel der früheren Studenten und Lehrlinge, die jetzt in ihrer Berufspraxis wenigstens einen Teil ihrer damaligen Erfahrungen umzusetzen versuchten. Es waren Rechtsanwälte, Gewerkschaftssekretäre, Arbeits- und Sozialrichter, die sich zusammenfanden, um Benachteiligten zu helfen. Zunächst begann es mit der - meist kostenlosen - Rechtsberatung für ausländische Arbeitnehmer. Denen war die politische Mitwirkung mangels Wahlrecht in Deutschland versagt, und auch in den Gewerkschaften fanden sie sich nicht zurecht. Die Beratungsgespräche brachten oft direkte Einblicke in die Verhältnisse im francistischen Spanien, in Salazars Portugal und dem Griechenland der Obristen. Nicht selten engagierten sich die deutschen Helfer dann auch in den ausländischen Kulturvereinen. Dies führte zu einer allgemeinen Internationalisierung des Horizonts und Kontakten mit ausländischen Gewerkschaften, wie sie keine Generation vorher gekannt hatte. Aber auch die Kontakte unter den deutschen Beteiligten wurden enger: Man veranstaltete gemeinsam Seminare zum Arbeits- und Sozialrecht, und man traf sich schließlich ganz regelmäßig auf örtlicher und regionaler Ebene. Da diese Richter und Anwälte häufig auch selbst Gewerkschaftsmitglieder waren, entstanden sog. gewerkschaftliche Arbeitskreise meist im Rahmen der ÖTV. Es ging dabei aber nicht um Gewerkschaftspolitik, sondern um den fachlichen Meinungsaustausch und natürlich auch um Personalpolitik. Etliche dieser Zirkel, die vielfach freundschaftlichen Charakter annahmen, bestehen bis heute. Es waren dennoch beileibe keine Privatveranstaltungen, sondern öffentliche Foren, zu denen in den Hochschulen, Gerichten und Gewerkschaften am Ort allgemein eingeladen wurde.

Diese neue, sich als progressiv aus dem Geist von 1968 empfindende arbeitsrechtliche Öffentlichkeit beeinflusste auch die Betriebsräte in Schulungen nach dem Betriebsverfassungsgesetz und gewann Einblicke in die praktische Arbeitswelt.

17 BAG (v. 22.12.1980) AP Nr. 70 zu Art. 9 GG Arbeitskampf. 
Ich selbst war 1978 an die Akademie der Arbeit in der Universität Frankfurt auf die Stelle für Arbeitsrecht, Verfassungsrecht und Politikwissenschaft berufen worden und habe die Herausbildung dieser fachbezogenen Öffentlichkeit miterlebt. Sie war zwar inhaltlich und personell begrenzt, aber über das ganze Land verbreitet und deshalb einflussreich. Die Richter, Anwälte und Gewerkschaftssekretäre verstanden das Arbeitsrecht als Arbeitnehmerschutzrecht, setzten die Gleichbehandlung und insbesondere die Entgeltgleichheit auch im Tarifrecht durch und vertraten die Wirksamkeit der Grundrechte im Arbeitsrecht. Natürlich marschierten sie dann auch durch die Institutionen bis zum BVerfG und zum EuGH. Die Offenheit des gesetzlichen Arbeitsrechts, die Unvollkommenheit vieler Tarifverträge und schließlich das Richterrecht des Arbeitskampfs ermöglichte diesem Teil der 68er-Generation also eine beträchtliche anhaltende Wirkung. Dabei hat auch die beratende Tätigkeit dieser Juristengeneration in den Gewerkschaften (und über das Wirtschaftswissenschaftliche Institut des DGB) dazu beigetragen, dass man das Arbeitsrecht dort nunmehr als ein Instrument begreifen konnte, über welches man demokratisch verfügen konnte und verfügen wollte.

\section{E. Neuorientierung in der Arbeitsrechtsdogmatik}

Diese Wirkung beruhte vor allem darauf, dass ihr eine moderne und praxisbetonte Rechtsdogmatik zugrunde lag. Nicht zuletzt unter Rückbesinnung auf die Startphase des deutschen Arbeitsrechts unter Lotmar und Sinzheimer ${ }^{18}$ rekapitulierte man die Grundfragen der Arbeitsbedingungen. Auszugehen war vom Tarifvertrag als Instrument gesellschaftlich-normativer Selbststeuerung. Fast jeder Tarifvertrag enthielt dabei immer auch die faktische Anerkennung des privaten Unternehmertums. Er bestätigte damit indirekt den marktförmigen Funktionstypus der Arbeitswelt. In der Garantie der Koalitionsfreiheit erkannte man die Tarifautonomie als ein System „kollektiv ausgeübter Privatautonomie“. Das BAG verwendet diese Rechtsfigur bis heute, um die normative Wirkung tariflicher Regelungen zu erklären.

Entscheidend war jedoch, dass die Funktionsfähigkeit dieser kollektiven Privatautonomie Verfahrensabsicherungen voraussetzte, die einen fairen sozialen Ausgleich zwischen den so unterschiedlichen Arbeitsmarktparteien ermöglichten.

Das war der Hintergrund für die Einschränkung der Aussperrung auf streikbezogene Abwehrkampfmaßnahmen durch das BAG von 1980 sowie für die Erkenntnis des BVerfG von 1991, wonach Streik, Aussperrung und Tarifautonomie in Art. 9 Abs. 3 GG grundrechtlich garantiert sind. Im Zentrum dieser Entscheidung stehen die Sätze: „Tarifautonomie ist darauf angelegt, die strukturelle Unterlegenheit der einzelnen Arbeitnehmer beim Abschluss von Arbeitsverträgen durch kollektives Handeln auszugleichen und damit ein annähernd gleichgewichtiges Aushandeln der Löhne und Arbeitsbedingungen zu ermöglichen. Soweit Arbeitskämpfe zu einem Ungleichgewicht führen, wird die Funktionsfähigkeit der Tarifautonomie beeinträchtigt." "19

18 Vgl. dazu Martin Becker, Arbeitsvertrag und Arbeitsverhältnis in Deutschland. Vom Beginn der Industrialisierung bis zum Beginn des Kaiserreichs (= Studien zur europäischen Rechtsgeschichte 76), Frankfurt 1995.

19 BVerfGE (v. 26.6.1991) 84, 212, 229. 
Erinnert man sich demgegenüber an das BAG-Verdikt von $1955,{ }^{20}$ wonach ,Arbeitskämpfe im allgemeinen unerwünscht“ seien, weil sie den „sozialen Frieden stören“, dann ermisst man die Distanz bis zu ihrem rechtlichen Verständnis als Movens sozialer Sicherheit. Bis heute wird die BVerfG-Entscheidung übrigens von konservativer Seite angefeindet. ${ }^{21}$

Vorausgegangen war allerdings eine wesentliche Veränderung gewerkschaftlicher Streikaktionen. Das BAG hatte 1976 den kurzen verhandlungsbegleitenden Warnstreik wegen seines „,milden Drucks“ zugelassen und vom ultima-ratio-Prinzip ausgenommen. ${ }^{22}$ Daraufhin entwickelte zuerst die IG Metall ihre Streikstrategie der „Neuen Beweglichkeit". Sie bestand in einer zentral geplanten Folge vieler kurzer Warnstreiks in unterschiedlichen Betrieben und war für die Gewerkschaften billiger als die klassischen Erzwingungsstreiks. Bei Warnstreiks entfallen meistens Streikunterstützungszahlungen. Vor allem aber haben sie wegen ihres demonstrativen Charakters eine wesentlich intensivere Öffentlichkeitswirkung.

Darin lag nun wieder ein typischer 68er-Gedanke. Die damaligen Akteure hatten ihre Durchschlagskraft doch vor allem aus der demonstrativen „Herstellung von Öffentlichkeit" und ihrer Medienresonanz bezogen. Jetzt nahmen die Gewerkschaften diese Lehre auf, weil sie erkannten, dass Arbeitskämpfe künftig primär als Kämpfe um die öffentliche Meinung geführt werden müssten. 1984 bestätigte das BAG die neue Kampftaktik. ${ }^{23}$ Zwar wurde die Privilegierung des Warnstreiks $1988^{24}$ wieder zurückgenommen, doch ist diese Taktik wegen der gleichzeitigen Einschränkung des ultima-ratioPrinzips bis heute möglich und üblich.

Das grundrechtsgeleitete Konzept der Marktwirtschaft hatte auch im Individualrecht wichtige Konsequenzen. In seiner Handelsvertreter-Entscheidung erkannte das BVerfG,${ }^{25}$ dass der grundrechtsgebundene Gesetzgeber die Pflicht habe, das Privatrecht durch zwingende Normen derart zu regeln, dass auch im privaten Individualvertrag keine unsoziale Ausbeutung des wirtschaftlich Schwächeren ermöglicht wird.

Wieder geht es um die aktive Rolle des Sozialstaats bei der Gestaltung privater Rechtsbeziehungen durch Verfahrensregelungen und materiellen Mindestschutz. In dieser grundrechtsgeleiteten Bändigung der sozialen Dysfunktionalitäten des liberalen Marktes durch Gegenmacht-Bildung und Freiheitsschutz kann man durchaus eine Konsequenz aus dem Kapitalismus-Verdacht, aber auch aus dem Demokratievertrauen der alten $68 \mathrm{er}$ sehen. ${ }^{26}$

20 Siehe Anm. 1.

21 Vgl. z.B. Reinhard Richardi, Arbeitsrecht als Teil freiheitlicher Ordnung. Von der Zwangsordnung im Arbeitsleben zur Arbeitsverfassung der Bundesrepublik Deutschland, BadenBaden 2002, 245, 262.

22 BAG (v. 17.12.1976) AP Nr. 51 zu Art. 9 GG Arbeitskampf.

23 BAG (v. 12.9.1984) AP Nr. 81 zu Art. 9 GG Arbeitskampf.

24 BAG (v. 21.6.1988) AP Nr. 108 zu Art. 9 GG Arbeitskampf.

25 BVerfGE (v. 7.2.1990) 81, 242.

26 Vgl. dagegen aber auch Andreas Fischer-Lescano, Kritik der praktischen Konkordanz, KJ 41 (2008), 226 m.w.N. 


\section{F. Die Rolle der Rechtswissenschaft}

Die neue Dogmatik ist vor allem von theoretisch engagierten Richtern durchgesetzt worden. An arbeitsverfassungsrechtlich führender Stelle wirkten Thomas Dieterich und Jürgen Kühling. Sie konnten dabei auf Vorarbeiten der Wissenschaft zum (kollektiven) Arbeitsrecht und zum Verfassungsrecht zurückgreifen. Die dafür notwendige rechtstheoretische Grundlagenforschung hatte u.a. Ulrike Wendeling-Schröder 1992 mit ihrer Habilitationsschrift ${ }^{27}$ vorgenommen. Insgesamt blieb die Rolle der universitären Arbeitsrechtler aber eigentümlich blass.

Das mag an der feindseligen Stellenbesetzungspolitik der alten Universitäten gegenüber progressiven Wissenschaftlern gelegen haben. Das notorisch konservativ verwurzelte „Arbeitsrechts-Kartell“ ${ }^{\prime 28}$ war unverändert abwehrbereit. Es hatte nur an einigen meist neueren Hochschulen Einbrüche hinnehmen müssen (Hamburg, Bremen, Hannover, Gießen, Frankfurt, Konstanz). Vielleicht scheuten die „Neuen“ gerade deshalb eine allzu deutliche Parteinahme für die Arbeitnehmerseite über persönliche Beziehungen hinaus. Dagegen hatten die alten Arbeitsrechtler keine Scheu vor deutlich arbeitgeberorientiertem Engagement. Vielleicht haben sich die Gewerkschaften damals auch zu sehr auf das ihnen näherstehende Praktiker-Milieu der schon erwähnten Arbeitskreise verlassen. Jedenfalls setzte sich die traditionell distanzierte Wissenschaftsposition gegenüber der Praxis fort. Der IG-Metall-Kongress zum Arbeitskampfrecht von 1973 ist also eher eine Ausnahme geblieben. Die Wissenschaft kompensierte diese verpassten Chancen jedoch durch eine verstärkt rechtssoziologische und übernationale Blickweise. ${ }^{29}$ Das wissenschaftliche Erbe von 1968 ist wohl eher hier zu suchen. Demgegenüber konnten die Gewerkschaften trotz wachsender europäischer Herausforderungen für ihre eigenen Spezialisten insgesamt wenige internationale Fortschritte erzielen.

\section{G. Fortwirkungen der Neuorientierung}

Dementsprechend sind die Fortwirkungen der Neuorientierung wiederum primär im richterlichen Arbeitsrecht zu suchen. Die nächstfolgende Generation hat die Position eines zweckbezogenen und grundrechtsstrukturierten Arbeitsmarktverständnisses tatsächlich fortgesetzt und ausgebaut. Trotz zunehmender Kritik von Arbeitgeberseite wurde die Zuständigkeit der Tarifparteien zur Regelung arbeitsbezogener Unterneh-

27 Ulrike Wendeling-Schröder, Autonomie im Arbeitsrecht. Möglichkeiten und Grenzen eigenverantwortlichen Handelns in der abhängigen Arbeit (= Juristische Abhandlungen 24), Frankfurt 1994.

28 So der Titel des provozierenden und entsprechend kritisierten Aufsatzes von Roderich Wahsner, Das Arbeitsrechtskartell - die Restauration des kapitalistischen Arbeitsrechtskartells in Westdeutschland nach 1945, KJ 7 (1974), 369.

29 Vgl. dazu etwa die Beiträge in: Thoma Klebe u.a. (Hg.), Recht und soziale Arbeitswelt, Festschrift für Wolfgang Däubler, Frankfurt 1999; Dieter Simon/Manfred Weiss (Hg.), Zur Autonomie des Individuums. Liber amicorum Spiros Simitis, Baden-Baden 2000; Armin Höland u.a. (Hg.), Arbeitnehmermitwirkung in einer sich globalisierenden Arbeitswelt. Liber amicorum Manfred Weiss, Berlin 2005. 
mensentscheidungen (Personalbemessung, betriebliche Mitbestimmung, Ladenöffnungszeiten) erweitert und damit auch Arbeitskämpfen geöffnet.

Letztes Beispiel ist die 2007 vom BAG bestätigte Erstreikbarkeit sog. SozialplanTarifverträge bei betrieblichen Standortveränderungen. ${ }^{30}$ Ähnliches gilt für das Arbeitskampfrecht. Die verbreitete Verselbständigung von Betriebsteilen zum Zweck der sog. Tarifflucht, d.h. des Verlassens bisheriger Tarifbindungen, hat das BAG arbeitskampfrechtlich aufzufangen versucht, indem es in solchen Fällen seit 2007 Sympathiestreiks zulässt. ${ }^{31}$ Dahinter steht noch immer das Konzept des gleichgewichtigen Aushandelns von Arbeitsbedingungen, auch wenn dies schon lange nicht mehr explizit sozialstaatlich begründet wird.

Erheblich verbessert worden ist ferner die demokratische Struktur innergewerkschaftlicher Willensbildung. Die Satzung der 2001 gegründeten Vereinten Dienstleistungsgewerkschaft (ver.di) überlässt vor allem die tarifpolitischen Entscheidungen konsequent den Mitgliedern und beschränkt den hauptamtlichen Apparat weitgehend auf die Ausführung und Gewerkschaftsverwaltung.

Dennoch hat sich das Arbeitsrecht insgesamt nicht vom allgemeinen gesellschaftlichen Trend abkoppeln können, welcher den Markt als sich selbst steuerndes System begreift, das ohne übergeordnete demokratische Eingriffe am besten aus sich heraus legitimiert wird. Vor allem die Europäische Gemeinschaft, deren ursprünglicher Zweck der Durchsetzung liberaler Wirtschaftsfreiheiten zur Herstellung gemeinsamer Märkte sich zunehmend verselbständigt, hat hieran mitgewirkt. Die diesjährigen Urteile des EuGH in den Sachen Laval, Viking und Rüffert bewerten unternehmerische Dienstleistungs- und Niederlassungsfreiheit des europäischen Primärrechts höher als die - national legitimierte - Tarifautonomie, der nur noch arbeitsrechtliche Mindestschutz-Aufgaben zugestanden werden. ${ }^{32}$ Dieser Sieg der liberalen Grundfreiheiten gegen das solidarische Koalitionsgrundrecht ist sicher nicht das Ende dieser Auseinandersetzung. Vielleicht haben wir uns in Deutschland aber in den letzten Jahren zu sehr mit dem Markt als Problemlösungsmechanismus beschäftigt und seine notwendige demokratische Infrastruktur darüber vernachlässigt. Dieser solidarische Aspekt ist den jüngeren Arbeitsrechtlern an den Universitäten jedenfalls seit längerem etwas aus dem Blick geraten. Ihnen geht es mehr um die Unternehmensstrukturen im internationalen Wettbewerb. Daran soll das Arbeitsrecht ausgerichtet werden. Die gesellschaftlichen Voraussetzungen der kollektiven Ebene entgehen diesen betriebswirtschaftlich orientierten Juristen zunehmend. Die Grundrechte gerinnen dabei zu normativen Management-Methoden. Dort entwickeln sie allerdings durchaus beachtliche Durchsetzungskraft. Vor allem in ihrer Erscheinungsform als Diskriminierungsverbote zur Förderung der Marktchancen beflügeln sie den europäischen und den nationalen Gesetzgeber nachhaltig.

Auch die beruflichen Ziele der Studierenden des Arbeitsrechts haben sich geändert. Das Ideal ist oft die Partnerschaft in einer wirtschaftsrechtlichen Großkanzlei. Demgegenüber sind Richteramt und Universitätskarriere mit materiellem Verzicht verbun-

30 BAG (v. 24.4.2007) NZA 2007, 987.

31 BAG (v. 7.3.2007) NZA 2007, 1055.

32 Vgl. dazu Eva Kocher, Kollektivverhandlungen und Tarifautonomie - welche Rolle spielt das europäische Recht?, AuR 56 (2008), 13, einerseits; Fritz Scharpf, Interview, Mitbestimmung 54 (2008), 18, andererseits. 
den. Diese Feststellungen sind keineswegs herablassend oder resignativ gemeint. Aus der 68er-Perspektive lässt sich eine gewisse Ironie aber nicht ganz unterdrücken. Freilich handelt es sich eher um Selbstironie. Die hochgemute Öffentlichkeit ist inzwischen längst einer neuen Privatheit gewichen. Deren aktuelle Gefährdung durch überbordende staatliche Informationskontrollen trägt nurmehr ihrerseits dazu bei, eine überraschende öffentliche Gegenbewegung hervorzurufen.

Erbschaften von 1968 spielen dabei jedoch kaum eine große Rolle. Verdeckt bleiben sie allerdings auch im Arbeitsrecht noch virulent. Sie sind dem allgemeinen Bewusstsein zwar weithin entglitten, doch bleiben sie ein nachweisbarer Teil unserer fachlichen Genetik. 